\title{
INCIDENCE AND MANAGEMENT OF TREATMENT-RELATED SIDE EFFECTS FOR PATIENTS RECEIVING PLATINUM THERAPY IN AN OUTPATIENT ONCOLOGY CLINIC
}

\author{
INDHUMATHI P*, SHANMUGA PRIYA E \\ Department of Pharmacy Practice, School of Pharmaceutical Sciences, Vels University, Pallavaram, Chennai, Tamil Nadu, India. \\ Email: indhumathi498@gmail.com
}

Received: 04 April 2017, Revised and Accepted: 20 April 2017

\section{ABSTRACT}

Objective: Platinum agents (PAs) are the most commonly used chemotherapeutic agents, aiming to induce tumor cell death by cytotoxicity. This leads to have high potential for toxicity and adverse effects. The main objectives of this study were to describe the incidence of side effects caused by platinum therapy and to conclude the management of treatment-related side effects for patients receiving platinum therapy.

Methods: A descriptive, retrospective study was carried out over a period of 9 months. Laboratory reports of 100 cancer patients were recorded. All statistical analyses were performed using SPSS 17.0 and GraphPad Prism. A p $<0.05$ (95\% confidence interval) was considered significantly throughout the study.

Results: Among 100 patients 55\% were male and 45\% were female. A total of $70 \%(\mathrm{n}=70)$ patients who received platinum compounds as chemotherapy regimen, among them $97.14 \%(n=68)$ patients appears to have anemia. Among the occurrence of adverse drug reactions, the incidence of anemia was found to be significant $(n=68,97.14 \%)$. Statistically significant difference was found in the incidence of anemia between PAs and nonPAs treated group $(\mathrm{p}=0.0001)$.

Conclusion: Among PAs cisplatin-induced anemia and nephrotoxicity appears to be high in this study. There is a need to improve the management of anemia induced by PAs. Since anemia is preventable, this study emphasizes the need to improve the management of anemia induced by PAs.

Keywords: Platinum agents, Cytotoxicity, Adverse effects, Anemia, Cisplatin.

(C) 2017 The Authors. Published by Innovare Academic Sciences Pvt Ltd. This is an open access article under the CC BY license (http://creativecommons. org/licenses/by/4. 0/) DOI: http://dx.doi.org/10.22159/ajpcr.2017.v10i8.18927

\section{INTRODUCTION}

The platinum derivatives such as cisplatin, carboplatin, and oxaliplatin are antineoplastic agents with remarkably useful in the treatment of several tumor types. Recognition of cisplatin's cytotoxic activity was the result of a serendipitous observation that bacterial growth in culture was altered when an electric current was delivered to the media through platinum electrodes. The growth, change was noted to be similar to that produced by alkylating agents and radiation. It was found that a platinum chloride complex, now known as cisplatin, generated by the current was responsible for the changes. Carboplatin is structurally similar to cisplatin in which the chloride groups of the parent compound are replaced by a carboxycyclobutane moiety. It shares a similar spectrum of clinical activity with cisplatin, and cross resistance is common. Oxaliplatin is an organoplatinum compound in which the platinum is complexed with an oxalate ligand as the leaving group and to diaminocyclohexane. Its spectrum of activity differs substantially from the other platinum compounds and includes notable activity against colorectal cancers [1,2].

Platinum compounds are used for the treatment of testicular cancer, ovarian cancer, bladder cancer, head and neck cancer, esophageal cancer, small and non-small cell lung cancer, breast cancer, cervical cancer, stomach and prostate cancers, as well as Hodgkin's and nonHodgkin's lymphomas, neuroblastoma, sarcomas, multiple myeloma, and melanoma [3]. The cytotoxicity of the platinum derivatives depends on platinum binding to DNA and the formation of intrastrand cross-links or adducts between neighboring guanines which lead to cellular damage by distorting the normal DNA conformation and preventing bases that are normally paired from lining up with each other. Interstrand cross-links also occur [4].
Platinum agents (PAs) leads to diverse side-effects and toxicities due to cytotoxicity or apoptosis induced in cancer cells. The general toxicity profile differs between the three platinum drugs [5]. Cisplatin is an antineoplastic agent, with high potential for producing serious toxicities such as nephrotoxicity, ototoxicity, and peripheral neuropathy. Carboplatin is potential to cause renal damage, peripheral neuropathy, ototoxicity, nausea, and vomiting are much less than that of comparable cisplatin doses. Oxaliplatin produces peripheral neuropathies and unique cold-induced neuropathies [1,2,6]. All of the platinum derivatives have significant potential to cause hypersensitivity reactions, including anaphylaxis $[7,8]$.

This current retrospective study was aimed to determine the incidence of treatment-related side effects in patients receiving platinum therapy and compare the effectiveness of existing management modalities in an outpatient oncology clinic.

\section{METHODS}

This retrospective observational study was carried out in an oncology department for 9 months from August 2015 to April 2016. The study protocol was approved by the Institutional Ethics Committee - IEC/ DOPV/2015/03 of Vels University. Consent from the hospital authorities and oncologists were obtained before accessing patient medical records. Biochemical parameters of cancer patients were recorded from the laboratory database.

\section{Inclusion criterion}

All cancer patients belonging to either gender or more than 18 years of age, who were receiving platinum compounds under any standard regimen, were included in the study. 


\section{Exclusion criterion}

Patients with documented pre-existing kidney disease, neurological disorder, history of allergic reactions to cisplatin or other platinumcontaining compounds, hearing impairment, and myelosuppression were excluded from the study.

\section{Statistical analysis}

The incidence of dichotomous categorical variables was analyzed by means of chi-square test to determine the presence or absence of statistically significant difference. All statistical analyses were performed using SPSS 17.0 and GraphPad Prism. A p $<0.05$ (95\% confidence interval) was considered significantly throughout the study.

\section{RESULTS}

Among 100 patients $55 \%$ were male, and $45 \%$ were female. The various indications were cervical cancer, ovarian cancer, testicular cancer, lung cancer, stomach cancer, esophageal cancer, breast cancer, and squamous cell carcinoma. The most common indication was carcinoma cervix (Table 1)

The majority of patients were in the age group of 50-59 years ( $n=32)$, $32 \%$ of patients was more prone to cancer, followed by $40-49$ years $(\mathrm{n}=24,24 \%)$ (Table 2).

The study population includes 100 cancer patients. Among them, $70(70 \%)$ patients were treated with platinum therapy, and $30(30 \%)$ were treated with non-platinum therapy (Table 3).

The most common PAs used in this study population were cisplatin 30 (30\%), carboplatin 25 (25\%), and oxaliplatin 15 (15\%) (Table 4).

Side effects in cancer patient after platinum therapy were found to be fatigue $20(28.5 \%)$, insomnia $7(10 \%)$, dysphagia $10(14.2 \%)$,

Table 1: Gender distribution

\begin{tabular}{ll}
\hline Gender & Number of patients $\mathbf{n = 1 0 0}(\mathbf{\%})$ \\
\hline Male & $55(55)$ \\
Female & $45(45)$ \\
\hline
\end{tabular}

Table 2: Age distribution

\begin{tabular}{ll}
\hline Age & Number of patients $\mathbf{n = 1 0 0}(\%)$ \\
\hline $19-29$ & $9(9)$ \\
$30-39$ & $16(16)$ \\
$40-49$ & $24(24)$ \\
$50-59$ & $32(32)$ \\
$60-69$ & $17(17)$ \\
$70-89$ & $2(2)$ \\
\hline
\end{tabular}

Table 3: Platinum versus non-platinum therapy

\begin{tabular}{ll}
\hline Therapy & Number of patients $\mathbf{n = 1 0 0}(\mathbf{\%})$ \\
\hline Platinum therapy & $70(70)$ \\
Non-platinum therapy & $30(30)$ \\
\hline
\end{tabular}

Table 4: PAs

\begin{tabular}{ll}
\hline Drugs & Number of patients $(\mathbf{n}=\mathbf{7 0})$ \\
\hline Cisplatin & 30 \\
Carboplatin & 15 \\
Oxaliplatin & 25 \\
\hline
\end{tabular}

PA: Platinum agents itching and redness $8(11.4 \%)$, bleeding 4 (5.7), constipation 2 (2.8\%), numbness (10\%), and vomiting 12 (17.14\%) (Table 5 and Fig. 1).

The occurrence of anemia was found to be high with cisplatin treatment when compared with other PAs (Table 6 and Fig. 2).

A total of $70 \%(\mathrm{n}=70)$ patients who received platinum compounds as chemotherapy regimen, among them $97.14 \%(n=68)$ patients appears to have anemia.

The incidence of anemia was compared between patients on PAs and non-PAs (NPA) using Chi-square test at $95 \% \mathrm{CI}$, and the result is graphically represented in Fig. 3.

The incidence of nephrotoxicity between PAs and NPAs was determined in cancer patients included in the study. The results are graphically represented in Fig. 4.

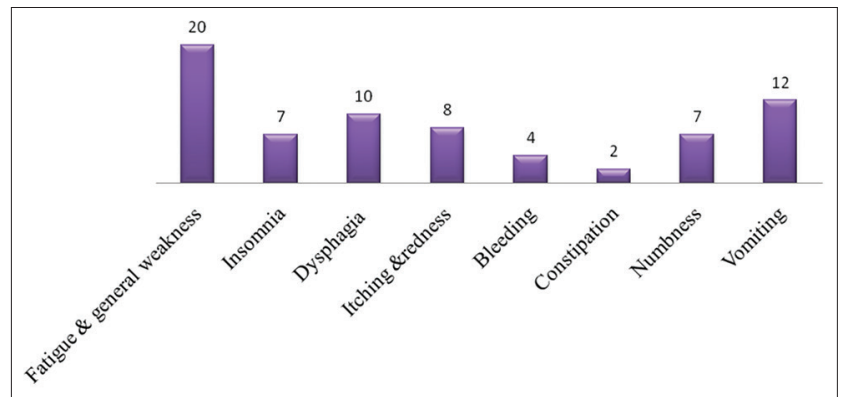

Fig. 1: Side effects after platinum therapy in cancer patients

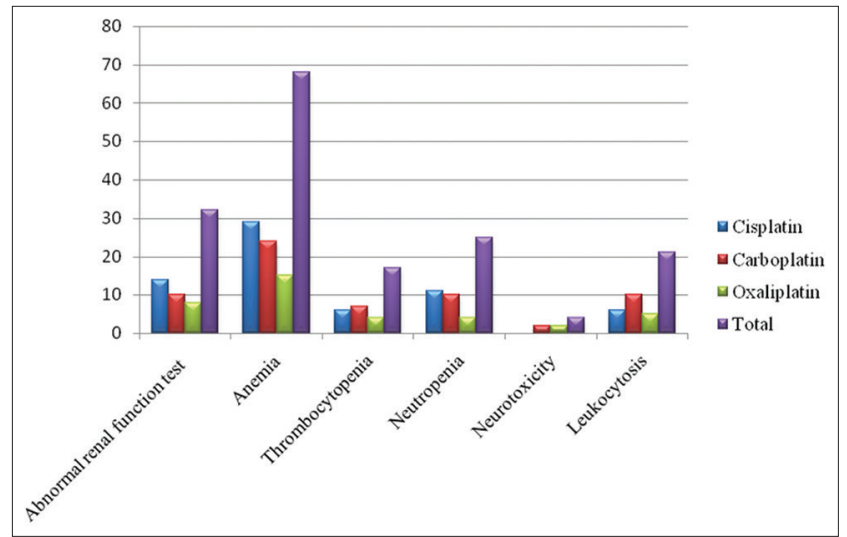

Fig. 2: Occurrence of adverse drug reactions after platinum therapy

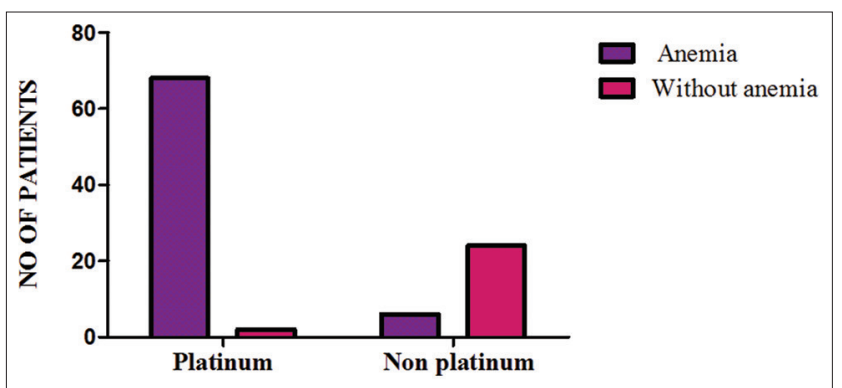

Fig. 3: Incidence of anemia between platinum agents (PA) and non-PAs treated groups $(p=0.0001$, odds ratio $=136.0$, relative risk=4. 857) 
Table 5: Side effects after platinum therapy in cancer patients

\begin{tabular}{lc}
\hline Side effects & Number of patients (\%) \\
\hline Fatigue & $20(28.5)$ \\
Insomnia & $7(10)$ \\
Dysphagia & $10(14.2)$ \\
Itching and redness & $8(11.4)$ \\
Bleeding & $4(5.7)$ \\
Constipation & $2(2.8)$ \\
Numbness & $7(10)$ \\
Vomiting & $12(17.14)$ \\
\hline
\end{tabular}

Table 6: Occurrence of ADR after platinum therapy

\begin{tabular}{lllll}
\hline ADR & Cisplatin (n=30) & Carboplatin (n=25) & Oxaliplatin (n=15) & Total number of patients (n=70) \\
\hline Abnormal renal function test & 14 & 10 & 8 & 32 \\
Anemia & 29 & 24 & 15 & 68 \\
Thrombocytopenia & 6 & 7 & 4 & 17 \\
Neutropenia & 11 & 10 & 4 & 25 \\
Neurotoxicity & - & 2 & 2 & 4 \\
Leukocytosis & 6 & 10 & 5 & 21 \\
\hline
\end{tabular}

ADR: Adverse drug reactions

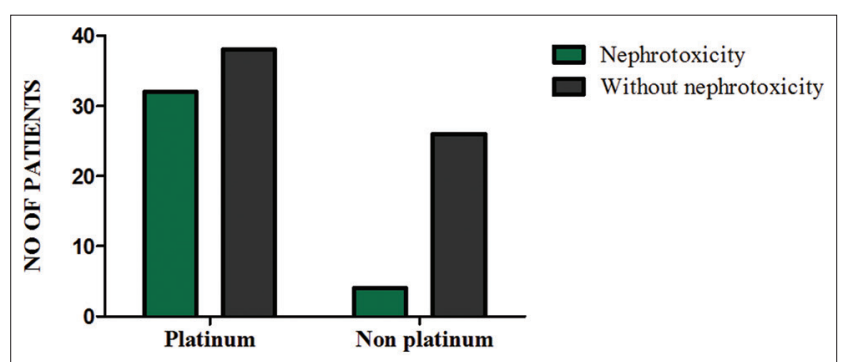

Fig. 4: Incidence of nephrotoxicity between platinum agents (PA) and non-PAs treated groups $(\mathrm{p}=0.0064$, odds ratio $=4.632$, relative risk=2.971)

\section{DISCUSSION}

The occurrence of toxicities in patients with cancer chemotherapy is found to be grave concern, the elimination of side effects, and possible toxicities become a major problem during chemotherapy, especially with PAs.

During 9 months of this study, case reports of 100 patients were analyzed out of which $55(55 \%)$ were male and 45 (45\%) were female. Among them, the majority of cancer patients were in the age group of 50-59 (n=32, 32\%). Among 100 cancer patients, 70 (70\%) were treated with PAs cisplatin $30(30 \%)$, carboplatin $25(25 \%)$, and oxaliplatin $15(15 \%)$

In our study, the following side effects were found to be fatigue $20(28.5 \%)$, insomnia $7(10 \%)$, dysphagia 10 (14.2\%), itching and redness 8 (11.4\%), bleeding 4 (5.7), constipation 2 (2.8\%), numbness $(10 \%)$, and vomiting $12(17.14 \%)$. The incidence of vomiting was secondary to fatigue. These findings were contrasted to the study reported by Swathi et al., 2015 and Laura et al., $2013[9,10]$.

Among the occurrence of adverse drug reactions (ADRs), the incidence of anemia was found to be significant $(n=68,97.14 \%)$. 97.14\% patients experienced anemia in the PA-treated group, whereas the incidence of anemia in NPA-treated group was found to be $20 \%$. Statistically significant difference was found in the incidence of anemia between PA and NPA-treated group ( $\mathrm{p}=0.0001)$. The occurrence of anemia was found to be high with cisplatin treatment when compared with other PAs $[9,11]$. Epoetin theta is a safe and effective treatment for anemia due to platinum-based chemotherapy in patients with solid tumors with a weekly starting dose of 20,000 IU. It is superior to blood transfusion [11].

Cisplatin induces nephrotoxicity characterized by acute and chronic renal insufficiency, renal magnesium wasting and electrolyte disturbances. $45.71 \%$ patients experienced nephrotoxicity in the PAtreated group, whereas the incidence of nephrotoxicity in NPA-treated group was found to be $13.33 \%$. A statistically significant difference was found in the incidence of nephrotoxicity between PA and NPA-treated group ( $\mathrm{p}=0.0064)[3,12]$.

Although nephrotoxicity was observed in the study population $(n=32,45.71 \%)$, the occurrence appears to be less when compared with anemia in this study $(n=68,97.14 \%)$. Frequency and severity of nephrotoxicity induced by platinum-based chemotherapy may be reduced by slow intravenous electrolyte infusions and maintaining the hydration before, during and immediately after the administration of PAs, especially cisplatin [12].

The other common adverse effect is myelosuppression characterized by neutropenia $(n=25,35.17 \%)$ and thrombocytopenia $(n=17$, $32.85 \%$ ). The reporting of ADRs has become an important component of monitoring and evaluation activities performed in hospitals [13]. The results of this study highlight the importance of monitoring the incidence of side effects in patients on platinum chemotherapy. The early detection and prompt management of these ADRs can reduce its health-related and economic effects on the patients [14].

\section{CONCLUSION}

Platinum-based chemotherapy is associated with increased the prevalence of toxicities and adverse effects. Among PAs cisplatininduced anemia and nephrotoxicity appears to be high in this study. 97.1\% of cancer patients experienced anemia in the PA-treated group, and also $92.85 \%$ cancer patients under platinum-based chemotherapy are exposed to ADR. Carboplatin-induced myelosuppression was found to be neutropenia (35.71\%), thrombocytopenia (32.85\%), and leucopenia $(45.71 \%)$. There is a need to improve the management of adverse effects. Since anemia is preventable, this study emphasizes the need to improve the management of anemia induced by PAs.

\section{REFERENCES}

1. Colvin M. Alkylating agents and platinum antitumor compounds. In: Kufe DW, Pollock RE, Weichselbaum RR, Bast RC, Gansler TS, Holland JF, et al., editors. Cancer Medicine. $6^{\text {th }}$ ed. Hamilton, Ontario: 
BC Decker; 2003. p. 759-79.

2. Guminski AD, Harnett PR, deFazio A. Scientists and clinicians test their metal-back to the future with platinum compounds. Lancet Oncol 2002;3(5):312-8

3. Florea AM, Büsselberg D. Cisplatin as an antitumor drug: Cellular mechanisms of activity, Drug resistance and induced side effects. Cancers (Basel) 2011;3(1):1351-71.

4. O'Dwyer PJ, Stevenson JP, Johnson SW. Clinical pharmacokinetics and administration of established platinum drugs. Drugs 2000;59 Suppl 4:19-27.

5. McWhinney SR, Goldberg RM, McLeod HL. Platinum neurotoxicity pharmacogenetics. Mol Cancer Ther 2009;8(1):10-6.

6. Andre T, Boni C, Mounedji-Boudiaf L, Navarro M, Tabernero J, Hickish T, et al. Oxaliplatin, fluorouracil, and leucovorin as adjuvant treatment for colon cancer. N Engl J Med 2004;350(23):2343-51.

7. Lazo JS. Bleomycin. Cancer Chemother Biol Response Modif 1999;18:39-45.

8. Sakaeda T, Kadoyama K, Yabuuchi H, Niijima S, Seki K, Shiraishi Y, et al. Platinum agent-induced hypersensitivity reactions: Data mining of the public version of the FDA adverse event reporting system, AERS. Int J Med Sci 2011;8(4):332-8
9. Swathi B, Bhavika D, Begum N. Adverse drug reaction profiles of commonly used platinum compounds in cancer chemotherapy. Int $\mathrm{J}$ Basic Clin Pharmacol 2015;4(2):284-9.

10. Astolfi L, Ghiselli S, Guaran V, Chicca M, Simoni E, Olivetto E, et al. Correlation of adverse effects of cisplatin administration in patients affected by solid tumours: A retrospective evaluation. Oncol Rep 2013;29(4):1285-92.

11. Tjulandin SA, Bias P, Elsässer R, Gertz B, Kohler E, Buchner A, et al. Epoetin theta in anaemic cancer patients receiving platinumbased chemotherapy: A randomised controlled trial. Arch Drug Inf 2010;3(3):45-53.

12. Arunkumar PA, Viswanatha GL, Radheshyam N, Mukund H, Belliyappa MS. Science behind cisplatin-induced nephrotoxicity in humans: A clinical study. Asian Pac J Trop Biomed 2012;2(8):640-4.

13. Tenti E, Gardin IC, Frassineti L, Amador D, Minguzzi M. Increase of reports of suspected adverse drug reactions in oncology. Int J Pharm Pharm Sci 2014;6(9):95-6.

14. Antony A, Joel J, Shetty J, Umar NF. Identification and analysis of adverse drug reactions associated with cancer chemotherapy in hospitalized patients. Int J Pharm Pharm Sci 2016;8(7):448-51. 